\title{
Technology for automatically characterizing the primary colors of the image and counting the number of objects
}

\author{
M I Khotilin ${ }^{1}$, R A Paringer ${ }^{1}$, I A Rytsarev ${ }^{1}$ and N S Kravtsova ${ }^{1}$ \\ ${ }^{1}$ Samara National Research University, Moskovskoe Shosse 34, Samara, Russia, 443086
}

\begin{abstract}
This work is devoted to the analysis of images and finding objects located on them, with the subsequent calculation of their number. The method of preprocessing based on, socalled, allocation of priority colors of the image is considered. The paper reflects the results of a study of the developed algorithm for object selection and clustering using priority image color analysis.
\end{abstract}

\section{Introduction}

Images have always occupied an important place in people's lives: whether they are rock carvings in primitive society, paintings of great artists and especially photographs With the advent of social networks, photographs began to move from the personal category to the public domain and became not only a new way of self-presentation, but also gave rise to other ways of thinking and seeing [1]. People literally translate their "I": they express their opinion, download the so-called "selfies", arrange discussions, share impressions and emotions in comments to photos or by color processing of photos with the help of various editors or filters.

The color characteristic is one of the most significant characteristics, which describes an image. With the help of it you can extract a variety of information that can be useful in many areas of human activity, such as psychology and sociology. In 2015, the Laboratory for the Study of Big Data, under the leadership of Lev Manovich, collected and analyzed 2.3 million photographs of major cities such as New York, Tokyo, Bangkok, San Francisco and eleven other global cities from the social network Instagram. With the help of the laboratory's analysis, we were able to see the "biological rhythms" by which the metropolis lives, and also how the life of a resident of one city differs from that of another, how time frames differ, etc. [2].

Also, the extracted data can be useful for example in medicine, in the selection and recognition of objects and in many other areas.

Recognition and selection of objects on images are one of the most significant tasks of computer vision and image processing. It finds its solution, for example, in finding various kinds of tumors in medicine, tracking objects in security systems, restoring images, and many others. To select objects of the image, various methods are used, both classical and modern, which use neural networks and large data processing tools, or BigData.

In this paper, we describe in detail the method of preprocessing an image by highlighting priority colors with their subsequent clustering. This preprocessing allows you to simplify the process of selecting image objects. 


\section{Highlighting the priority colors of the image}

What is an image? Each image can be viewed as a scene, which is a set of objects of different colors and sizes. Whatever we explore: a frame from a computer's webcam, or a high-resolution photo taken with a digital camera - any digital images are a collection of dots, which called "pixels". The method of selecting priority colors is based on working with pixels.

The essence of this method is as follows: since any digital image is a set of pixels characterized by their color brightness values, the entire image is treated as a three-layer table, each layer of which is a table with brightnesses of red, green and blue. Next, there is a color distribution analysis of each layer, which results in a corrected table (matrix) of the color. The next stage of the resulting new three-layer matrix is the resulting image. At this stage, the preprocessing ends.

Further, after preprocessing, the process of selecting image objects takes place. It consists in the passage of the entire image by a window of a fixed size, which makes it possible to determine the closedness of the color boundary. By determining the closedness of the color boundary, it is possible to determine various image objects.

At the moment, the development process is underway, with the purpose of further refinement of the method of selecting objects for working with large amounts of data.

We demonstrate the operation of the preprocessing method described above with an example. Consider the image shown in Figure 1.

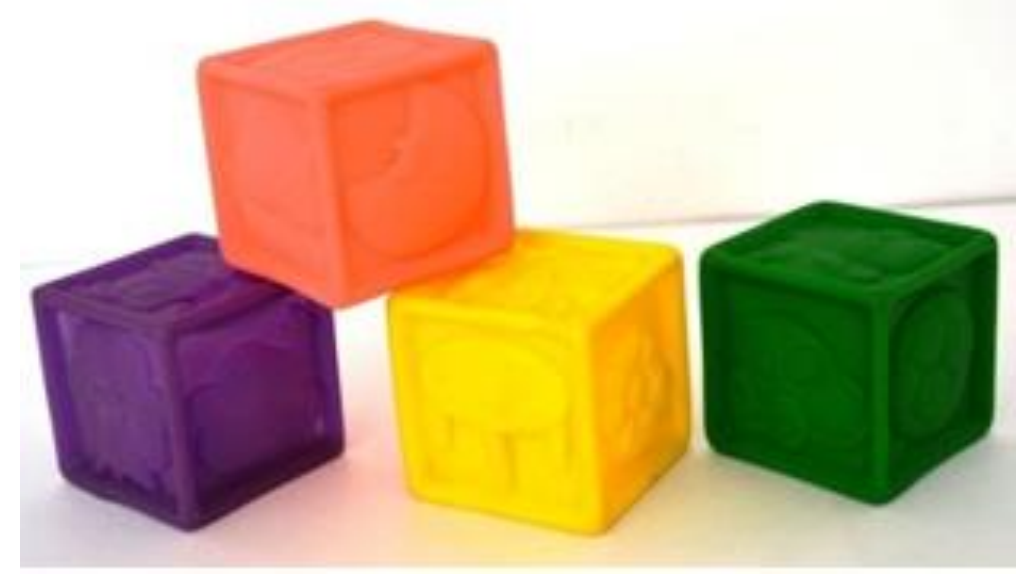

Figure 1. The original image.

In this image, each object has a certain color, and the relief of the object is highlighted by changing the intensity of the color. This image allows us to demonstrate all the steps of the algorithm.

Suppose that each object is uniquely determined by its colors - the main feature by which it can be designated. For the further analysis, a software package MATLAB R2014a was used to solve the problems of technical calculations.

Each pixel of the image in the RGB palette is represented as a blend of three colors: red, green and blue, the first letters of which bear the name of the palette. The distributions of the brightness of pixels on the main colors of the palette are shown in Figure 2.

However, the such distributions are not suitable for implementing the process of determining the priority colors of the image, due to the large number of extremums of these functions.

To solve this problem, it is necessary to bring the distributions to the peak type indicated in Figure 3.

The next step is the application of the method of mean values to color distributions. During the application of this method, the entire color distribution is divided into intervals, and the brightness values are summed. Next, changing the brightness in the original color table of the image, we get the resulting image, shown in Figure 4.

The main colors that occupy the largest area of the object of the resulting image are shown in Table 1. 
a)

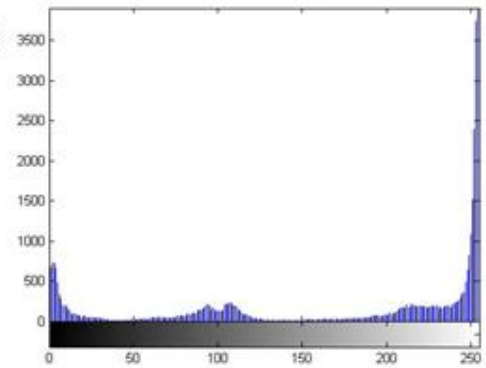

b)

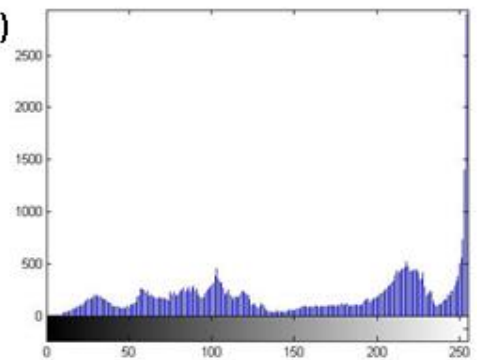

c)

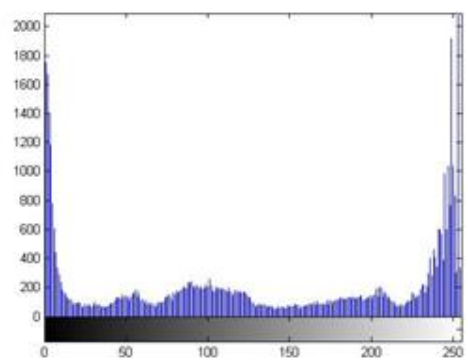

Figure 2. The distribution of the brightness of the pixels of the original image: a) red; b) green; c) blue.

a)

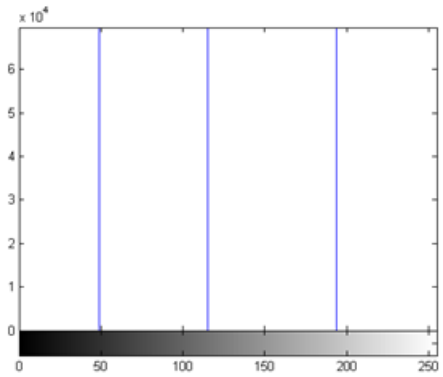

b)

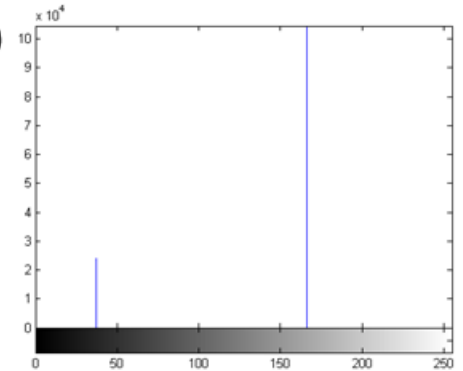

c)

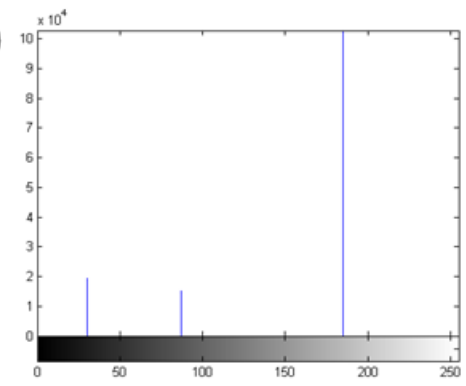

Figure 3. Resulting histograms of transformations.

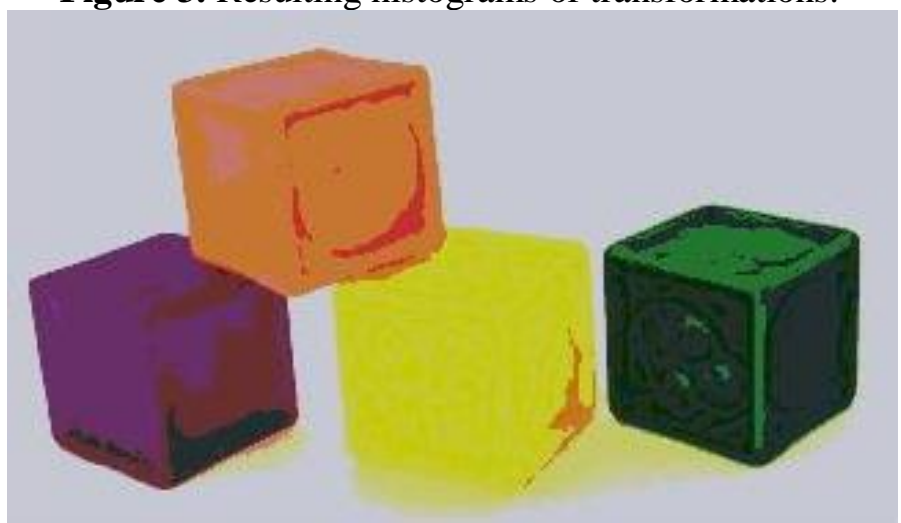

Figure 4. Resulting image. 
Table 1. The main colors of the image.

\begin{tabular}{|c|c|}
\hline & R: 200 \\
G: 117 \\
B:51 \\
\hline
\end{tabular}

After completing this set of operations, it was decided to use a more saturated image with a large number of constituent colors. The new original image is shown in Figure 5.

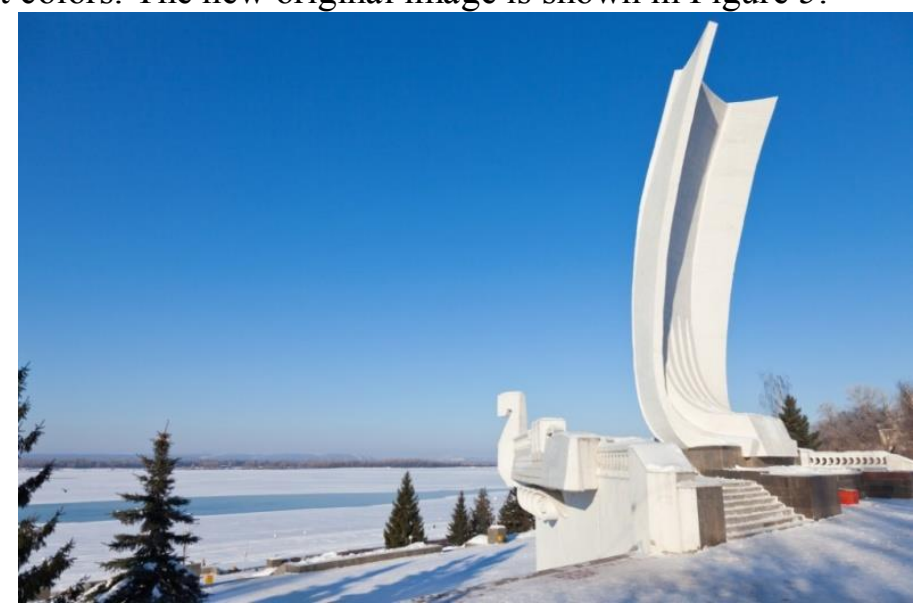

Figure 5. New original image.
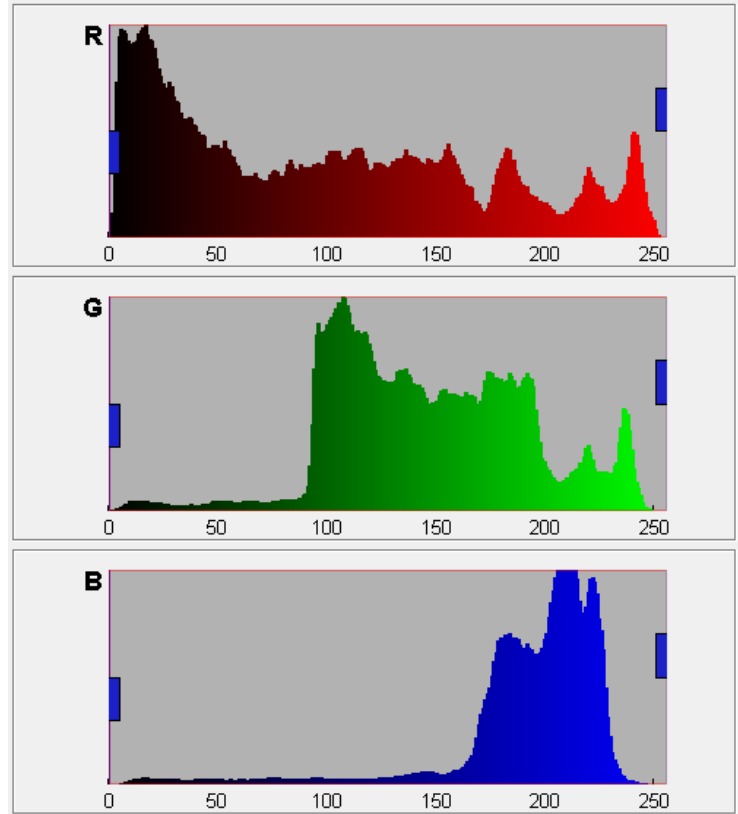

Figure 6. Color distribution of the original image. 
The brightness distribution of the original image is shown in Figure 6. The result of passing the image through all stages of the algorithm is the resulting image, shown in Figure 7. The main colors that occupy the largest area of the object of the resulting image are shown in Table 2.

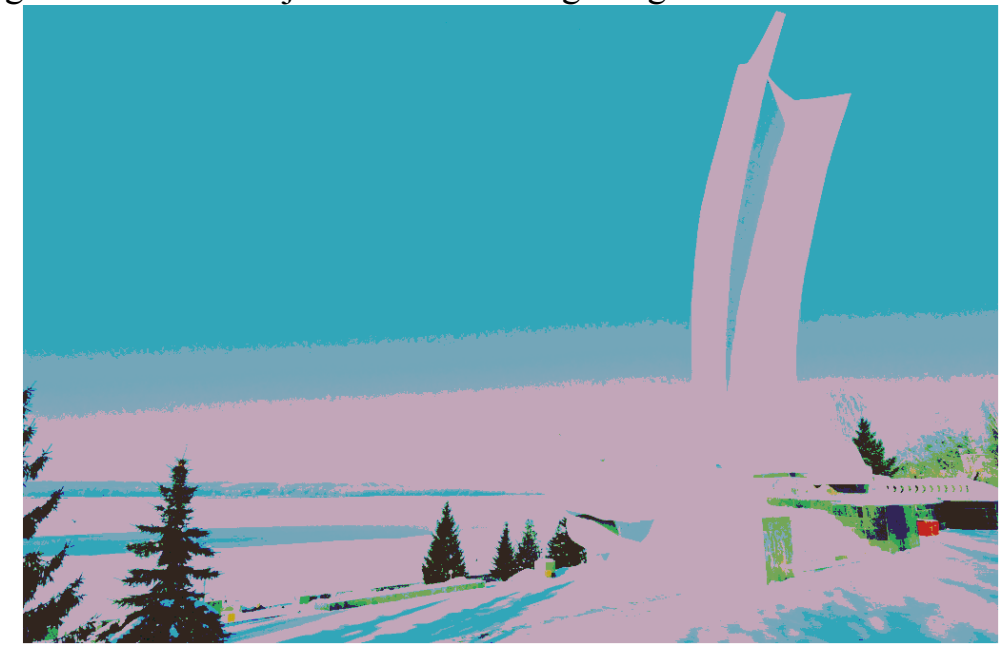

Figure 7. New resulting image.

Table 2. The main colors of the image.

\begin{tabular}{|c|c|}
\hline & R: 49 \\
G: 166 \\
B: 186 \\
\hline
\end{tabular}

\section{Conclusion}

Recognizing and highlighting key priority colors on images is one of the most significant tasks of computer vision and image processing. It finds its solution in various spheres of human activity. Often, existing methods of image processing and selection of objects on them, work well with relatively small amounts of raw data. Processing of large arrays of source images takes considerable time, which is absolutely unacceptable in a number of problems.

Currently, the authors of this article are working on the adaptation of the above methods to work with large amounts of data or BigData, and sre developing a software tool that allows appropriate processing of image arrays and recognition of objects on them. Also, the authors are completing the algorithm for recognizing image objects for using it in further work with the above technologies.

\section{References}

[1] Manovich L Z 2015 Instagram is a window in the mind and imagination of a person (MediaProfi)

[2] Khaykin S 2008 Neural networks: full course (Williams Publishing House) p 1131

[3] Sergeev V V 1998 Application of the methodology of pattern recognition in digital image processing tasks Autometry 2 63-76 
[4] Verhagen K, Doyne R and Groon F 1985 Pattern Recognition: Status and Prospects (Moscow: $\mathrm{R} \& \mathrm{~S}) \mathrm{p} 104$

[5] The use of neural networks in image recognition (Access mode: https://geektimes.ru/post/ 74326) (15.10.2017)

[6] Samal D I 2002 Algorithms for human identification from a photo portrait based on geometric transformations Thesis for the degree of Candidate of Science. Institute of Technical Sciences. cybern. NAS of Belarus (Minsk) p 170

[7] Graphic filters based on the torsion matrix (Access mode: https://habrahabr.ru/post/43895) (01.11.2017)

[8] Kravtsova N S, Paringer R A and Kupriyanov A V 2017 Parallel implementation of the informative areas generation method in the spatial spectrum domain Computer Optics 41(4) 585-587 DOI: 10.18287/2412-6179-2017-41-4-585-587

[9] Khotilin M and Blagov A 2016 Visualisation and cluster analysis of social networks CEUR Workshop Proceedings 1638 843-850

\section{Acknowledgment}

This work was partially supported by the Ministry of education and science of the Russian Federation in the framework of the implementation of the Program of increasing the competitiveness of Samara University among the world's leading scientific and educational centers for 2013-2020 years; by the Russian Foundation for Basic Research grants (\# 15-29-03823, \# 16-41-630761, \# 17-01-00972, \# 1837-00418), in the framework of the state task \#0026-2018-0102 "Optoinformation technologies for obtaining and processing hyperspectral data". 